\title{
PLAN URBANO-AMBIENTAL SANTA CATALINA, CORRIENTES
}

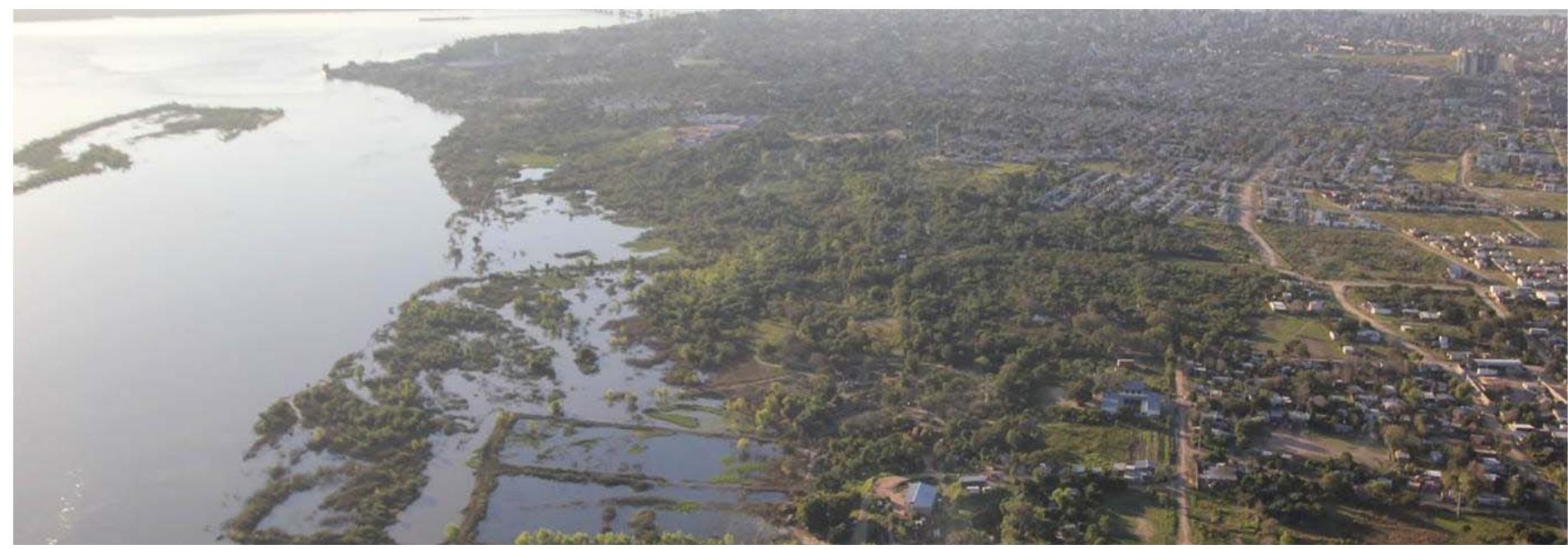




\section{RESUMEN}

En el año 2010, el Municipio de Corrientes gestionó la adquisición del predio de Santa Catalina al Ejército Argentino, en el marco de su política de desarrollo urbano. Concretado ese objetivo, en el año 2012, inició la elaboración del Plan Urbano Ambiental, desarrollado por un equipo de consultores externos y locales. ${ }^{1}$ Aprobado por el Concejo Deliberante (diciembre de 2013), actualmente el plan está siendo implementado por un fideicomiso creado al efecto. El objeto de este artículo es presentar los antecedentes que impulsaron la decisión política, el proyecto, sus objetivos, condicionamientos, estrategias; el proceso para su implementación, y reflexionar sobre logros y desafíos.

\section{ABSTRACT}

In the year 2010 the Municipality of Corrientes managed the acquisition of the land of Santa Catalina to the Argentine Army, in the frame of its urban development policy. In the year 2012, there initiated the production of the Environmental Urban Plan developed by an equipment of external and local consultants. Approved by the City Council (December 2013), nowadays the plan is being implemented by a Trust created for the effect. The purpose of this article is to present the background that prompted the political decision; the project, its objectives, constraints, strategies, the process for its implementation; and to reflect on achievements and challenges. Approved by the City Council (December 2013), nowadays the plan is being implemented by a Trust created for the effect. The purpose of this article is to present the background that prompted the political decision; the project, its objectives, constraints, strategies, the process for its implementation; and to reflect on achievements and challenges.

\section{JULIETA REPETTO}

Arquitecta, UNNE. Magíster en Antropología Social, UNAM. Directora General de Programas y Proyectos del Municipio de Corrientes (2009-2013) y directora del Plan Santa Catalina.

\section{LORENA SÁNCHEZ}

Arquitecta, UNNE. Magíster en Políticas Sociales, UNAM. Directora General de Ordenamiento Territorial del Municipio de Corrientes (2009-2013) y directora del Plan Santa Catalina.

\section{LAURA ALCALÁ}

Arquitecta, UNNE. Doctora en Urbanismo, UPC. Investigadora Adjunta CONICET. Consultora Local del Equipo de Dirección del Plan Santa Catalina (Consultoría CONICET 3291/13).

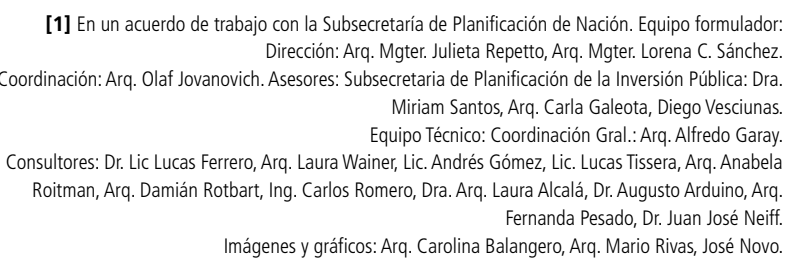


"... la ciudad de Corrientes siguió un proceso radio-concéntrico de crecimiento. Debido a límites naturales y urbanísticos muy fuertes..."

\section{DE "CAMPO DE MANIOBRAS" DEL EJÉRCITO A TIERRA DEL MUNICIPIO DE LA CIUDAD DE CORRIENTES}

\section{El contexto socio-urbano y político de la compra del Predio Santa Catalina}

La compra del predio Santa Catalina, de 2387 ha, por parte del Municipio de la Ciudad de Corrientes al Ejército Argentino, debe ser contextualizada en el marco del diagnóstico que hizo el Municipio sobre la ciudad y sobre su propia capacidad para incidir en el desarrollo de esta hacia los escenarios deseables, pero también en el marco de un contexto político e institucional favorable.

Desde su fundación el 3 de abril de 1588, la ciudad de Corrientes siguió un proceso radio-concéntrico de crecimiento. Debido a límites naturales y urbanísticos muy fuertes: el río Paraná al norte y al oeste, el aeropuerto al nordeste y las tierras del ejército al sur, la ciudad se extendió en abanico hacia el este, apoyándose en las principales vías de comunicación. En este proceso de crecimiento, alcanzó y traspasó el límite constituido por la ruta nacional N. ${ }^{\circ} 12$ al este de la urbanización, y avanzó sobre suelos ambientalmente frágiles y de interés paisajístico.

En Corrientes, los procesos de ocupación y urbanización del suelo seguidos fueron fundamentalmente impulsados por "el mercado" (principal tenedor del suelo urbano), subsidiariamente por "el Estado", principalmente a través de operaciones de vivienda del Instituto de Vivienda de Corrientes (INVICO) sobre suelo ofertado por el primero, y por "la necesidad", a partir de procesos informales de población sin posibilidades de acceso por ninguna de las vías anteriores.

En los últimos treinta años se acentuaron tres fenómenos, que en conjunto determinan una ciudad con altos desequilibrios e inequidades y progresiva segregación social: la presión por permisos de mayor edificabilidad en el área central fuertemente comprometida por las características de su trama fundacional; el consumo de suelo extensivo y de baja densidad en localizaciones cada vez más desprovistas en virtud del alza de los precios y el inicio de la construcción de clubes de campo en la zona este de la ciudad, de paisajes lacustres - como señalamos anteriormente- o hacia Santa Ana, localidad cercana que ofrece una conexión relativamente rápida a través de la ruta nacional $N .^{\circ} 12$.

El municipio tuvo escaso margen de incidencia sobre estos fenómenos. Más allá de la regulación y aplicación de la nor-

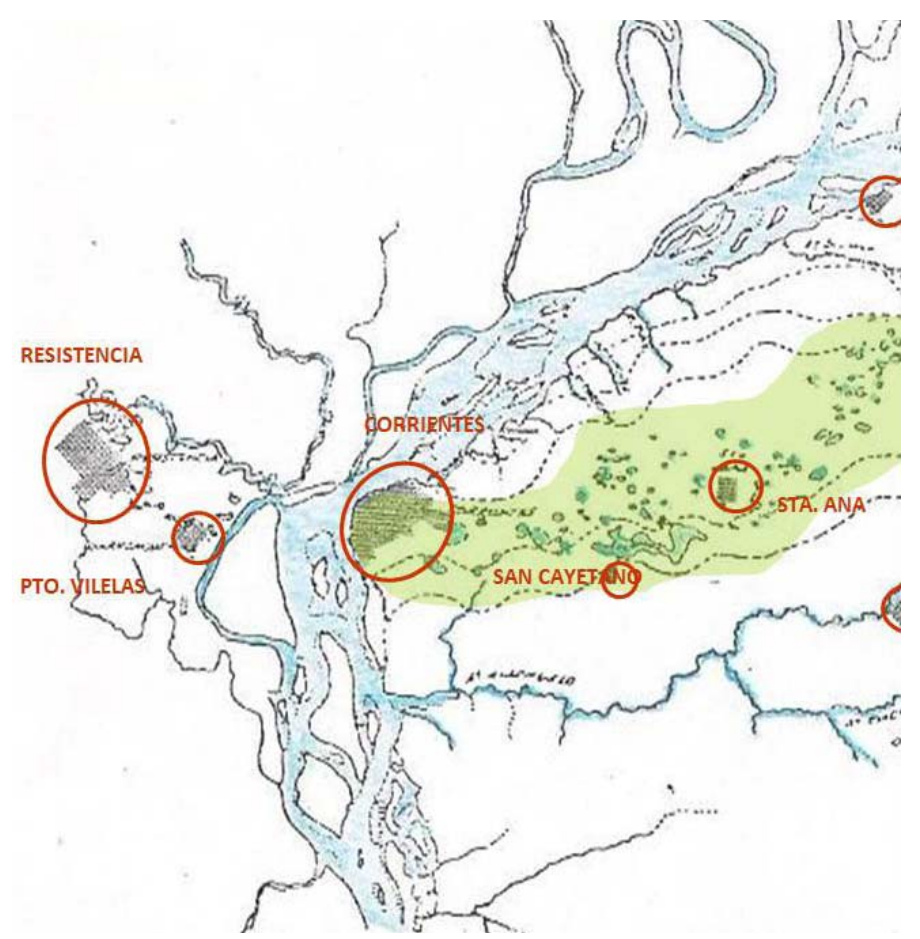

[Imagen 1. Paisaje lacustre de interés ambiental amenazado por la urbanización. Fuente: Municipio de la ciudad de Corrientes. Sin referencia bibliográfica]

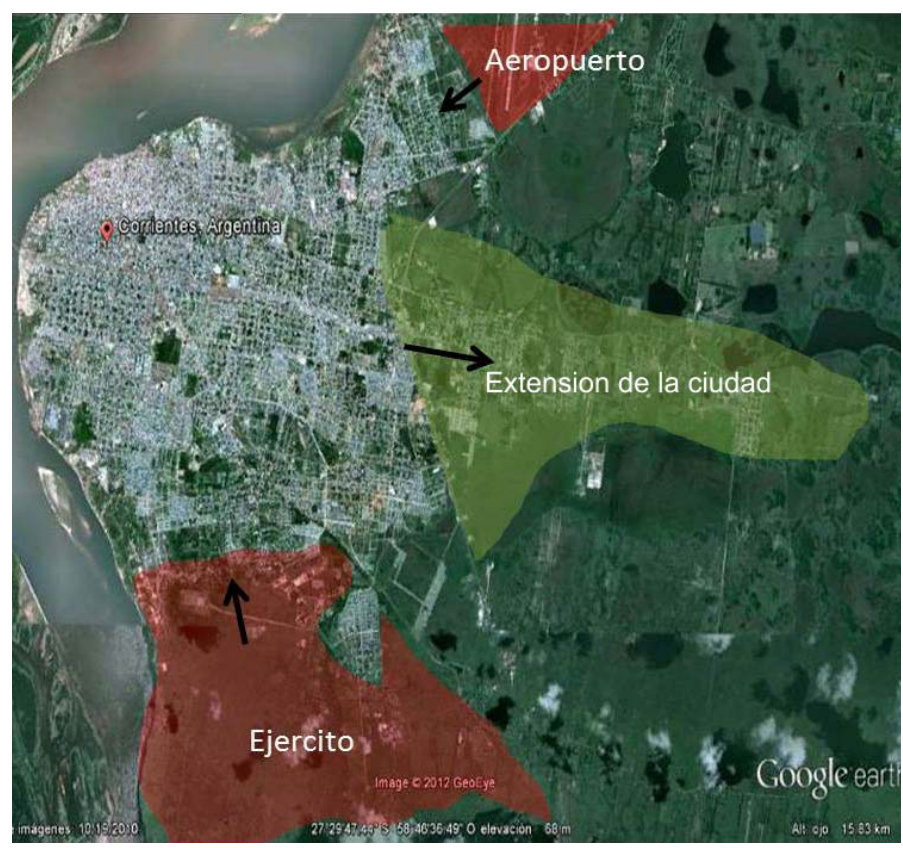

[Imagen 2. Proceso de expansión de la ciudad de Corrientes y principales condicionantes (o límites) del crecimiento (aeropuerto, ruta 12, tierras del ejército. Fuente: elaboración propia sobre la base de Google Earth] 
"La adquisición fue entendida por la actual gestión del intendente Fabián Ríos como una herramienta estratégica para conducir una expansión gradual y ordenada que reequilibre los procesos vigentes hacia un suelo apto..."

mativa urbana, careció de poder real y de herramientas eficaces para revertir, equilibrar y reorientar estos procesos.

En la última década, en el marco de ese contexto urbano y de importantes determinaciones a nivel del Estado Nacional de impulsar acciones de planificación y ordenamiento territorial, como lo son la puesta en marcha del Plan Estratégico Territorial (PET), la oferta de financiamiento para el fortalecimiento de la capacidad técnica de los gobiernos locales para la elaboración y gestión de nuevas políticas urbanas, así como el reemplazo de la ONABE por la Agencia de Administración de los Bienes del Estado, ${ }^{2}$ que tienen en conjunto la finalidad de facilitar procesos orientados a dotar de racionalidad y equidad distributiva la construcción del territorio, el Municipio de la Ciudad de Corrientes tomó la decisión política de adquirir los predios de Santa Catalina.

La adquisición fue entendida por la actual gestión del intendente Fabián Ríos como una herramienta estratégica para conducir una expansión gradual y ordenada que reequilibre los procesos vigentes hacia un suelo apto para estos fines; para operar sobre el mercado suelo, incidiendo en su precio desde el doble rol del municipio como regulador y propietario de la tierra, y para promover modalidades de venta más asequibles e inclusivas.

\section{EL PREDIO SANTA CATALINA UBICACIÓN, ORIGEN Y ANTECEDENTES EN EL PLANEAMIENTO DE LA PRINCIPAL TIERRA VACANTE DE LA CIUDAD DE CORRIENTES}

Santa Catalina se ubica al suroeste de la ciudad, limitando al oeste con el río Paraná y al sur con el Riachuelo, zona de singular valor paisajístico. Posee la mayor superficie en terrenos altos, no afectados por las inundaciones del Paraná.

El predio presenta su principal vinculación con la trama existente por la Av. Maipú. Además, la presencia del recorrido de un electroducto con suelo libre a su paso, la continuación de las avenidas Paysandú e Igarzabal y algunas otras vialidades internas que, aun sin urbanizar, habilitan la posibilidad de potenciar la continuidad norte-sur y el enlace con la zona este de la ciudad.

En relación con su topografía y condiciones ambientales,

[2] ELa Agencia de Administración de los Bienes del Estado creada por Decreto Presidencial 1382/12, tiene como fin proponer las políticas, normas y procedimientos respecto de la utilización y disposición de los bienes del Estado Nacional.
Santa Catalina presenta una situación de privilegio con relación a otros predios, ya que su altura es igual y en algunas partes supera en tres metros la media de la ciudad. Tiene áreas de valor paisajístico no solo en el sector de borde suroeste, sino también a lo largo del arroyo Pirayuí, que lo atraviesa de norte a sur.

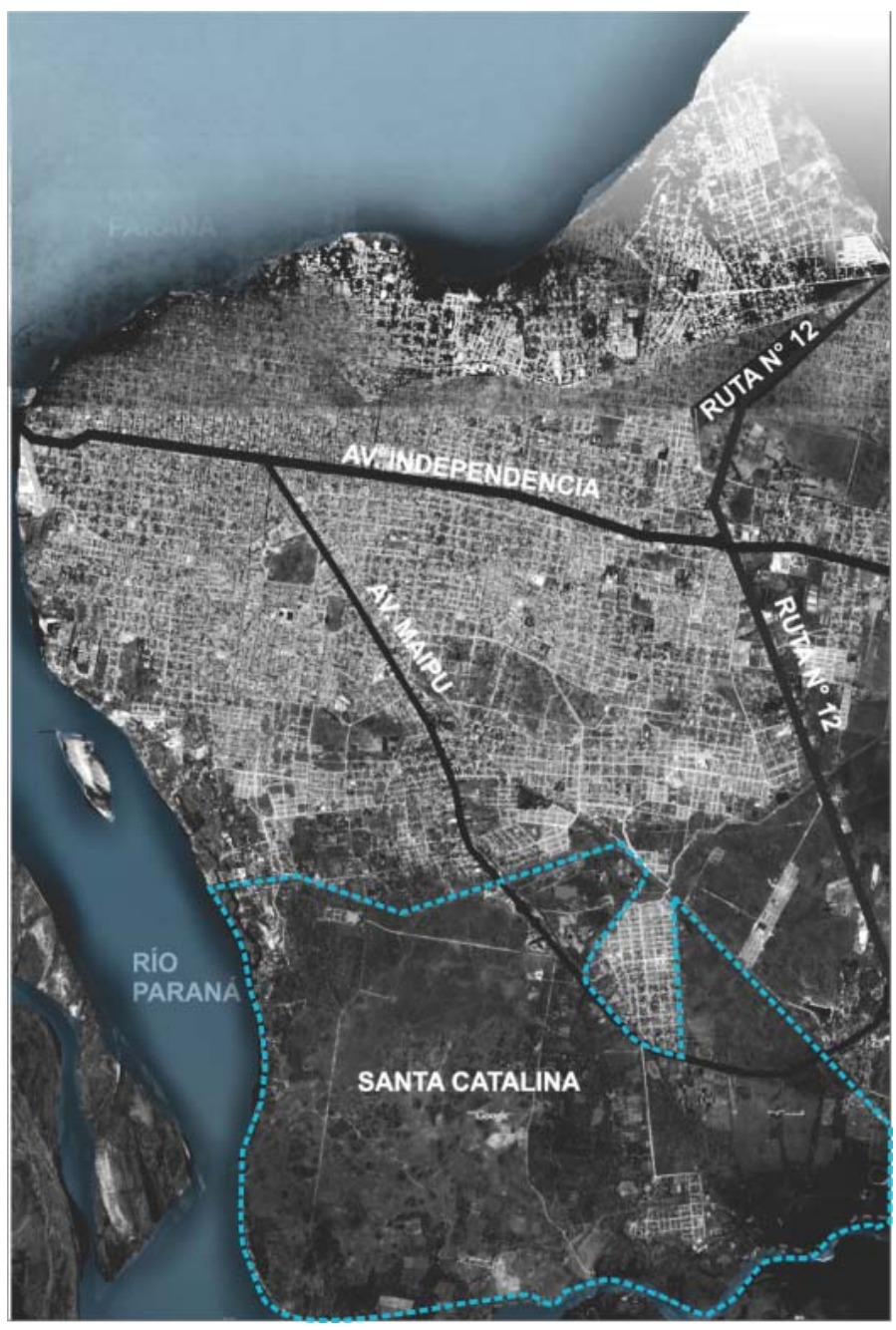

[Imagen 3. Santa Catalina y la ciudad de Corrientes. Fuente: elaboración propia sobre la base de Google Earth]

El predio Santa Catalina tuvo siempre un lugar en los distintos antecedentes de planeamiento de la ciudad de Corrientes. El Plan Roca (1950-1952) o el del arquitecto Cignoli (1967) le asignan una función centralmente ligada a la industria. El arquitecto Brian Thompson, al pensar en la conurbación Corrientes-Resistencia, lo integra como un espacio de continuidad en la expansión de la ciudad de Corrientes, con la impronta de plantear tempranamente, en ese momento, la idea de un segundo puente interprovincial. 
Ya en el año 1978, el Plan de Ordenamiento Urbano a cargo de la arquitecta Iris Bracco, que sienta las bases para el Código de Planeamiento de la Ciudad, plantea la continuidad de la Costanera existente como un desvío de tránsito pesado hacia el sur, proponiendo en Santa Catalina una estación de transferencia de cargas.

En el período 2006/2009, durante gestión del arquitecto Gallino Yanzi como secretario de Planificación, se elabora una propuesta sectorial para Santa Catalina, entendiendo que la ciudad tiene hacia el Sur el mejor escenario para su expansión. Se confeccionan dos propuestas, que no se conocieron públicamente; sin embargo, una de ellas tuvo como resultado tres proyectos de ordenanza que la configuran, según expediente N. 1119/S/09.

\section{EL PLAN URBANO AMBIENTAL SANTA CATALINA}

Como se puede observar, el Plan Urbano Ambiental Santa Catalina no comienza en 2012 con el equipo que elabora el masterplan, sino que es el resultado de numerosos estudios, de un proceso que se ha potencializado al recoger esa tradición y combinarla con nuevos enfoques que reinterpretan su función.

\section{Conformación delequipo deformulaciónymodalidad de trabajo}

En el marco del contexto político e institucional propicio antes mencionado, se firmó un Convenio entre el Municipio y la Subsecretaría de Planificación de la Nación, que permitió financiar un equipo de consultores especialistas en distintas disciplinas. La Dirección General del plan quedó a cargo del Municipio, y la Coordinación General fue compartida entre ambas instituciones. La Coordinación Técnica de la consultoría fue especialmente evaluada, y quedó a cargo del arquitecto Garay.

En esa evaluación, el Municipio tuvo en cuenta, entre otros aspectos, la escala y envergadura del proyecto, la necesidad de clarificar no solo un modelo físico de desarrollo del territorio, sino también - y fundamentalmente- un modelo de gestión económica y jurídica de la urbanización.

Teniendo en cuenta la necesidad de lograr una justa distancia objetiva de los intereses locales en pugna, pero con la clara decisión de desarrollar una idea genuina construida desde el conocimiento local del territorio, se propuso la Coordinación Técnica a un profesional con larga y probada trayectoria en este tipo de proyectos, ${ }^{3}$ y el equipo de consultores se constituyó con una mayoría de profesionales locales (Senior y Junior) elegidos por el Municipio según términos de referencia elaborados por él mismo y pautados con Nación, y algunos especialistas externos, seleccionados por la subsecretaría.

En virtud del convenio el proyecto se desarrolló en un plazo de doce meses. La modalidad de trabajo consistió en la realización de talleres con dos jornadas intensivas cada quince o veinte días. En ellos se producían los principales avances y lineamientos que conducían el trabajo posterior, continuado y sostenido por un intercambio on line entre los técnicos locales y los de Buenos Aires.

\section{Santa Catalina como herramienta de actuación}

Como resultado del análisis desarrollado durante la primera etapa de diagnóstico con una importante producción municipal, el equipo identificó una agenda de grandes cuestiones sobre las cuales la ciudad de Corrientes debería tomar algunas decisiones.

Los temas destacados fueron los siguientes:

1. Impacto de la urbanización sobre el medio físico y el soporte ambiental.

2. La complejidad del área central y la necesidad de una mayor descentralización.

3. Los desajustes que se producen en la transición entre el medio urbano y el rural.

4. El problema de la estructura vial, el tránsito y la movilidad. 5. La eficiencia de las infraestructuras y las condiciones de precariedad del hábitat.

Desdeesta perspectiva, el desarrolloconceptual dela operación debe poner en relación dos miradas: una desde los problemas de la ciudad (en términos de necesidad) y otra desde la perspectiva del municipio sobre el predio, en términos de posibilidad.

El resultado debe exponer cómo se puede contribuir a la solución de los cinco problemas que acaban de ser expuestos, poniendo en funcionamiento una institución que tenga la capacidad de poner en valor estos predios (Informe final Santa Catalina: 2013).

[3] Arquitecto (UBA) y máster en Urbanismo y Ordenamiento Territorial de la Universidad Libre de Bruselas, Bélgica. Ha recibido numerosos premios nacionales e internacionales, entre ellos, las Palmas Académicas del gobierno francés (1993), el Concurso Internacional para el desarrollo urbanístico de Santa María del Plata SA y el premio Nuevas Áreas Verdes para Puerto Madero-Revitalización y puesta en valor de la Costanera Sur (1996). Es profesor titular de la UBA, investigador del Instituto del Conurbano y responsable de la Carrera de Urbanismo de la Universidad Nacional de General Sarmiento. Ha sido profesor "Faculty Associate" en el Lincoln Institute of Land Policy Cambridge, Massachussetts, USA, y profesor titular en la Universidad Nacional Autónoma de México. Desarrolló proyectos urbanos en la Argentina y Chile. 


\section{Objetivos}

Los objetivos del proyecto se encuadraron en la idea de un modelo de ciudad deseable y de la especificidad del territorio que incorporar.

El primero se estructuró sobre la base de una serie de conceptos o ejes rectores que guiaron la propuesta:

- Mixtura social

- Mixtura funcional

- Máxima accesibilidad

- Máximo protagonismo del espacio público

- Permeabilidad hacia el río.

Estos ejes rectores se articularon y se pusieron en juego en la formulación a partir de los siguientes objetivos:

- Incorporar / recuperar un área importante por su localización y tendencia de crecimiento, actualmente sub-utilizada mediante una propuesta integral de urbanización, considerando necesidades a escala de la ciudad en su conjunto.

- Lograr una correcta vinculación del área con el resto de la ciudad consolidada.

- Posibilitar el acceso de la ciudad al río desde el punto de vista de sus actividades recreativas y productivas.

- Preservar las áreas y sitios de interés natural, paisajístico o turístico, teniendo en cuenta las necesidades actuales.

- Crear condiciones para la localización de actividades económico-productivas no admitidas en áreas centrales.

- Indicar proyectos particularizados de equipamientos e infraestructura de escala urbana-regional.

- Promover una distribución equilibrada de actividades residenciales y equipamiento barrial.

- Jerarquizar con claridad la red vial para inferir su correcto uso evitando concentraciones excesivas que generen problemas de tránsito.

\section{Condicionantes de diseño}

El territorio de Santa Catalina no solo fue analizado en sus dimensiones histórica, ambiental, económica-social y topográfica, sino que además se consideraron todos los proyectos que de una $u$ otra manera confluyen en el presente en ese espacio territorial, tales como: la alternativa de traza del segundo puente interprovincial, una planta de tratamiento cloacal ${ }^{4}$ y el proyecto de parque industrial ${ }^{5}$ que ya había sido desarrollado.

Otro tipo de condicionante, no menos importante, fue el de las ocupaciones que existían en el predio, para lo cual se hizo

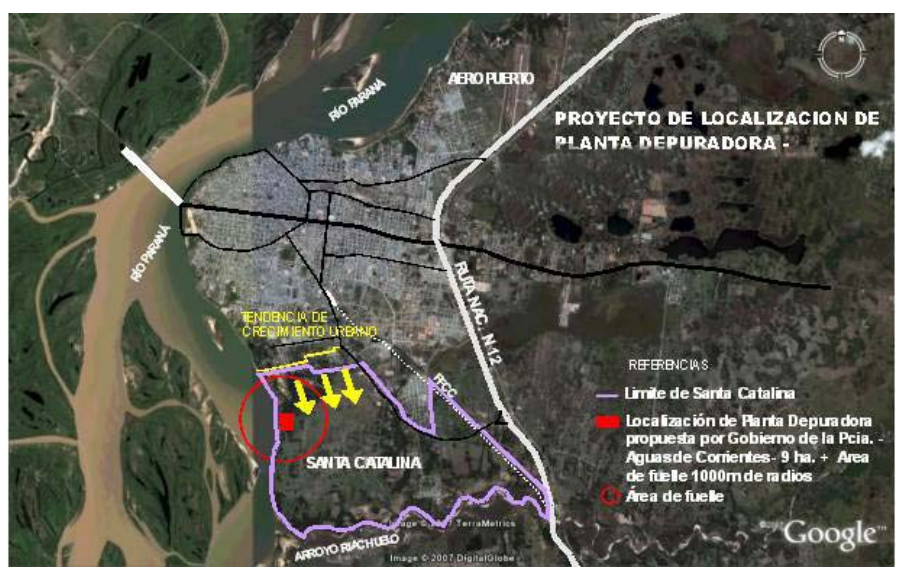

[Imagen 4. Ubicación del anteproyecto de planta de tratamiento de líquidos cloacales. Fuente: Municipio de la ciudad de Corrientes. DGOT]

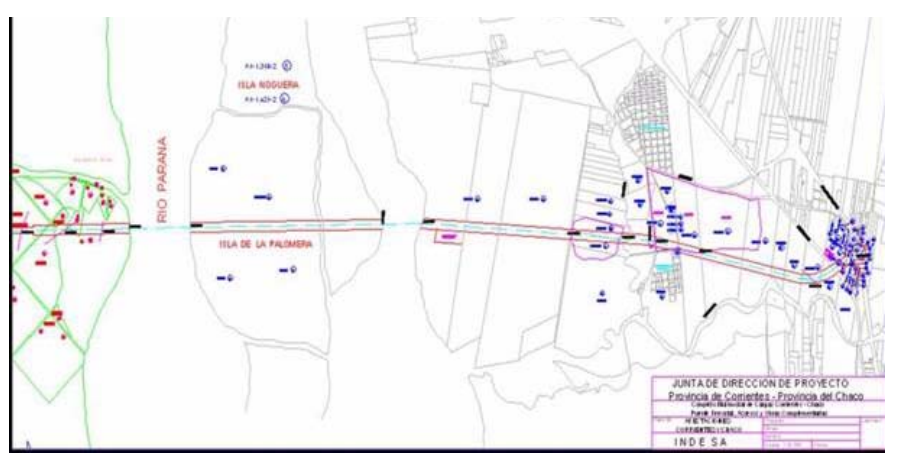

[Imagen 5. Anteproyecto traza prevista del segundo puente interprovincial. Fuente: Ing. Palmeyro]

un relevamiento de las situaciones de litigio y ocupación. Estas presentaban antinomias particulares: por una parte, pocas personas especulando/ litigando con prescripciones veinteañales en amplias superficies de suelo (aproximadamente 700has); y por la otra, muchas familias de escasos recursos económicos que viven en zonas inundables, en superficies pequeñas. 
"... el gran dilema en el proceso de análisis y diseño en Santa Catalina fue cómo potenciar sus condiciones físicas/ geopolíticas, incluir los megaproyectos para la ciudad y la región, y al mismo tiempo contrarrestar todas las posibles externalidades negativas que la propia ciudad podía depositar en este territorio".

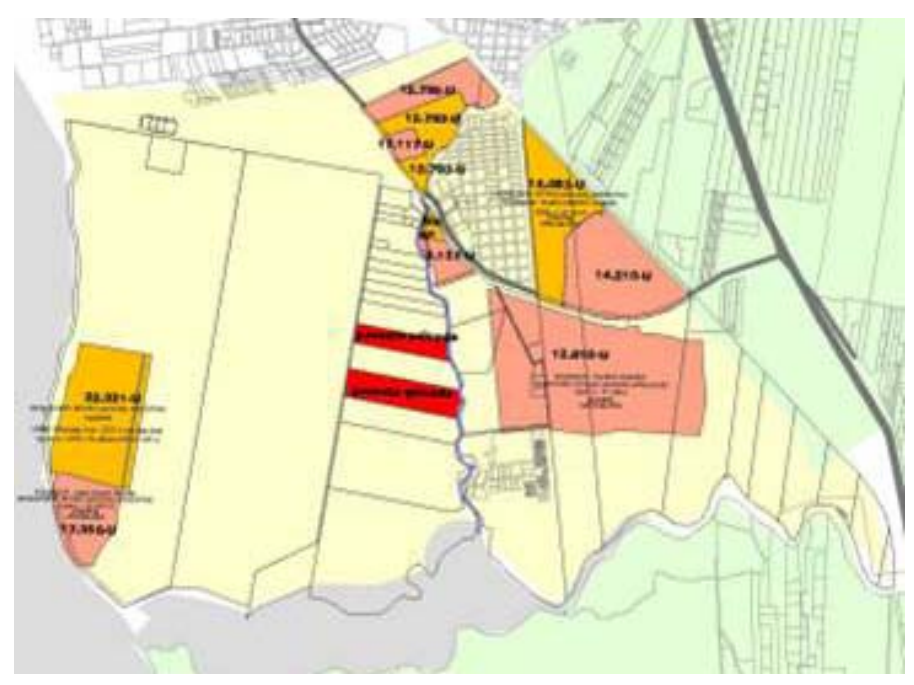

[Figura 6. Mapeo de prescripciones año 2013. Fuente: elaboración propia]

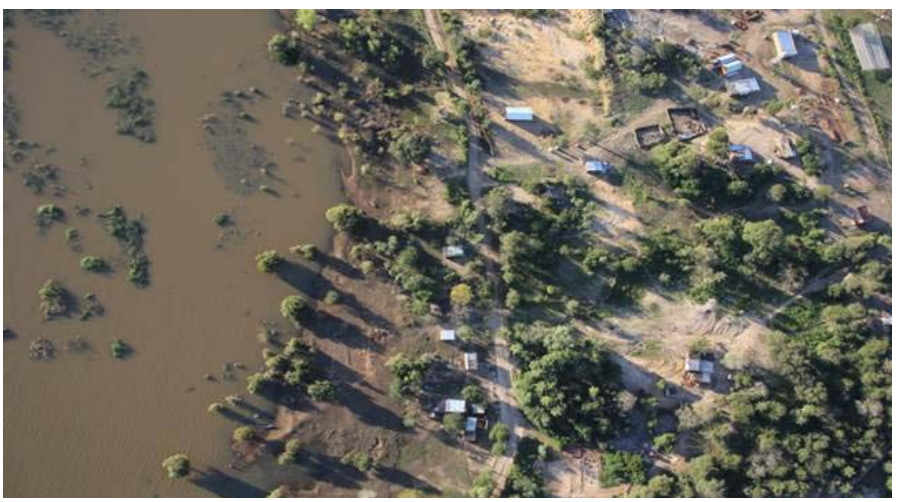

[Figura 7. Imagen fotográfica del sector de asentamientos en el bañado Sur. Foto: Edwin Harvey, contratado para el proyecto]

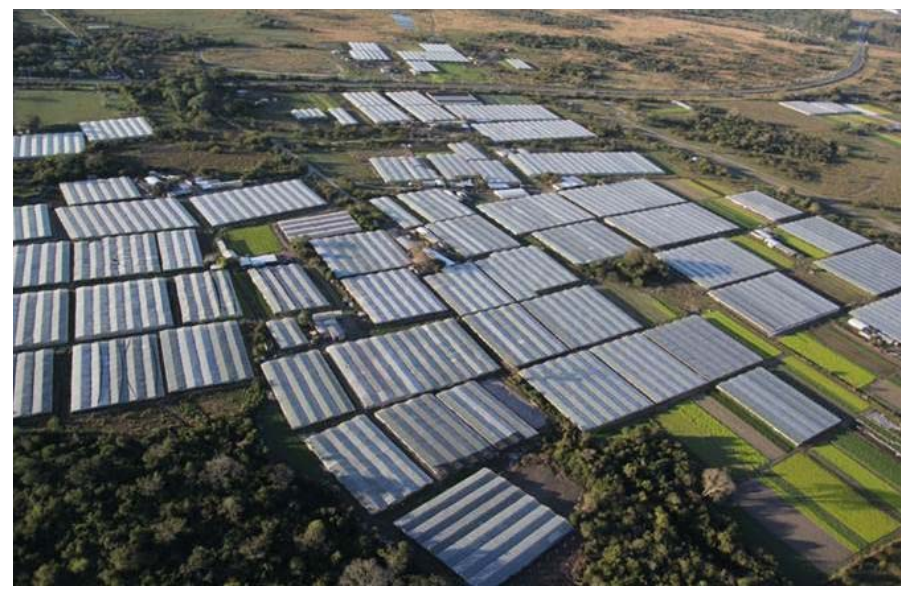

[Imagen 8. Imagen del sector de tendaleros, ubicados en una de las prescripciones. Actualmente el litigante alquila el sector a ciudadanos bolivianos que realizan cultivo intensivo. Foto: Edwin Harvey, contratado para el proyecto]

\section{Elementos estructurantes del diseño}

A partir de los distintos datos del terreno natural y construid y de los proyectos convergentes, el gran dilema en el proceso de análisis y diseño en Santa Catalina fue cómo potenciar sus condiciones fisicas/geopolíticas, incluir los megaproyectos para la ciudad y la región, y al mismo tiempo contrarrestar todas las posibles externalidades negativas que la propia ciudad podía depositar en este territorio.

\section{Estos elementos aportaron para establecer un primer despiece.}

1- Se marcaron los elementos estructurantes, como la previsión de la traza del segundo puente, la traza del electroducto y de los caminos existentes.

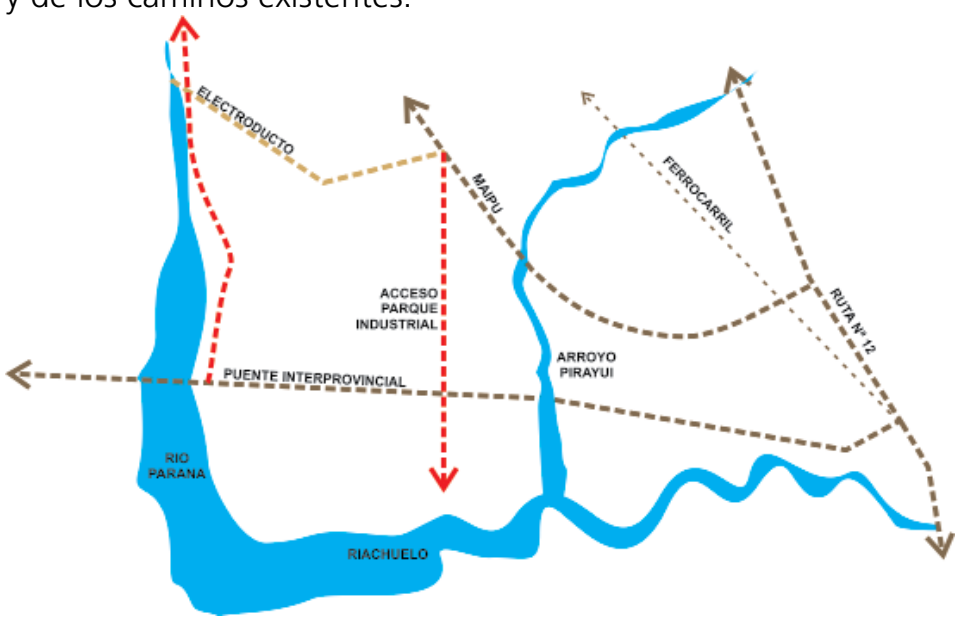

[Imagen 9. Esquema de los elementos estructurantes del trazado. Fuente: elaboración propia]

2- Las unidades de paisaje o conjunto de lugares o recintos cuyas condiciones ambientales o urbanas le confieren una identidad propia.

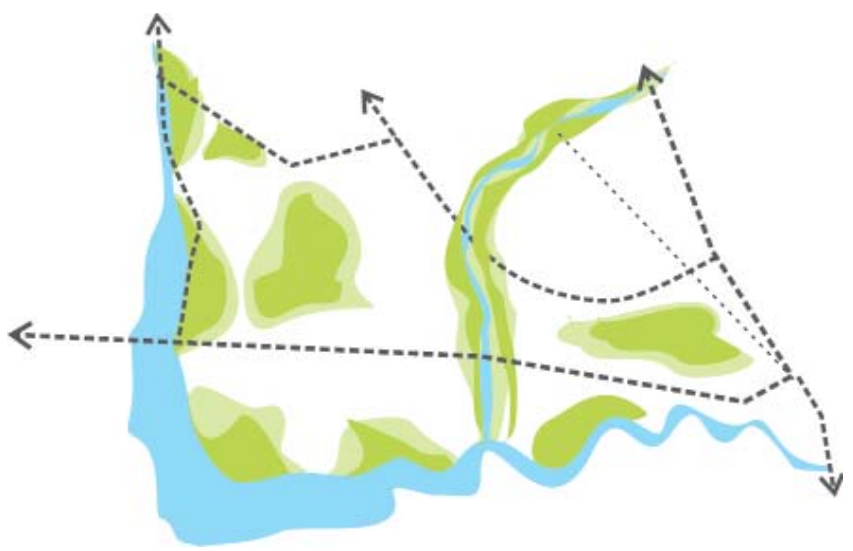

[Imagen 10. Esquema de las unidades ambientales/paisajísticas. Fuente: elaboración propia] 


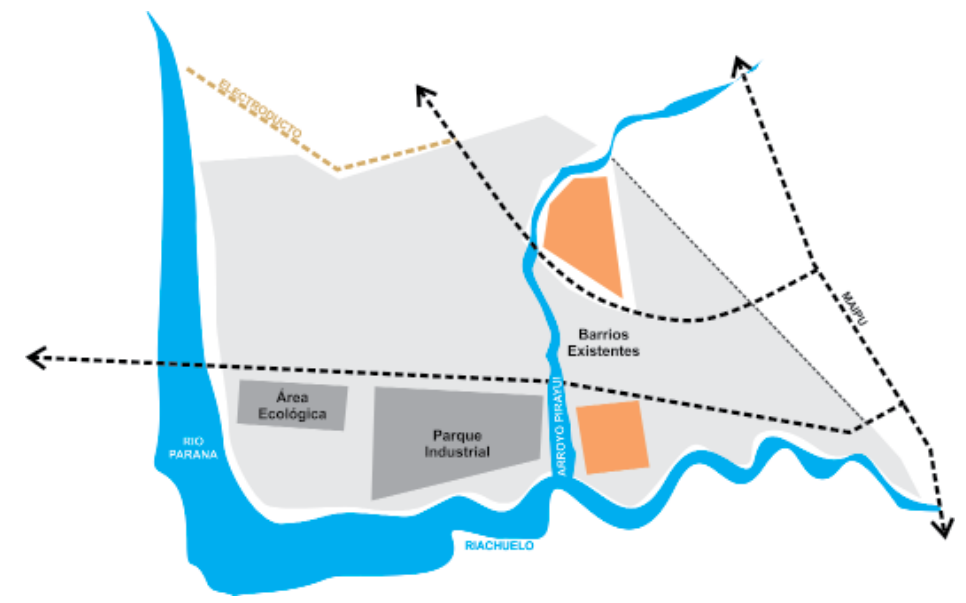

[Imagen 12. Esquema de urbanizaciones existentes y usos especiales. Fuente: elaboración propia]
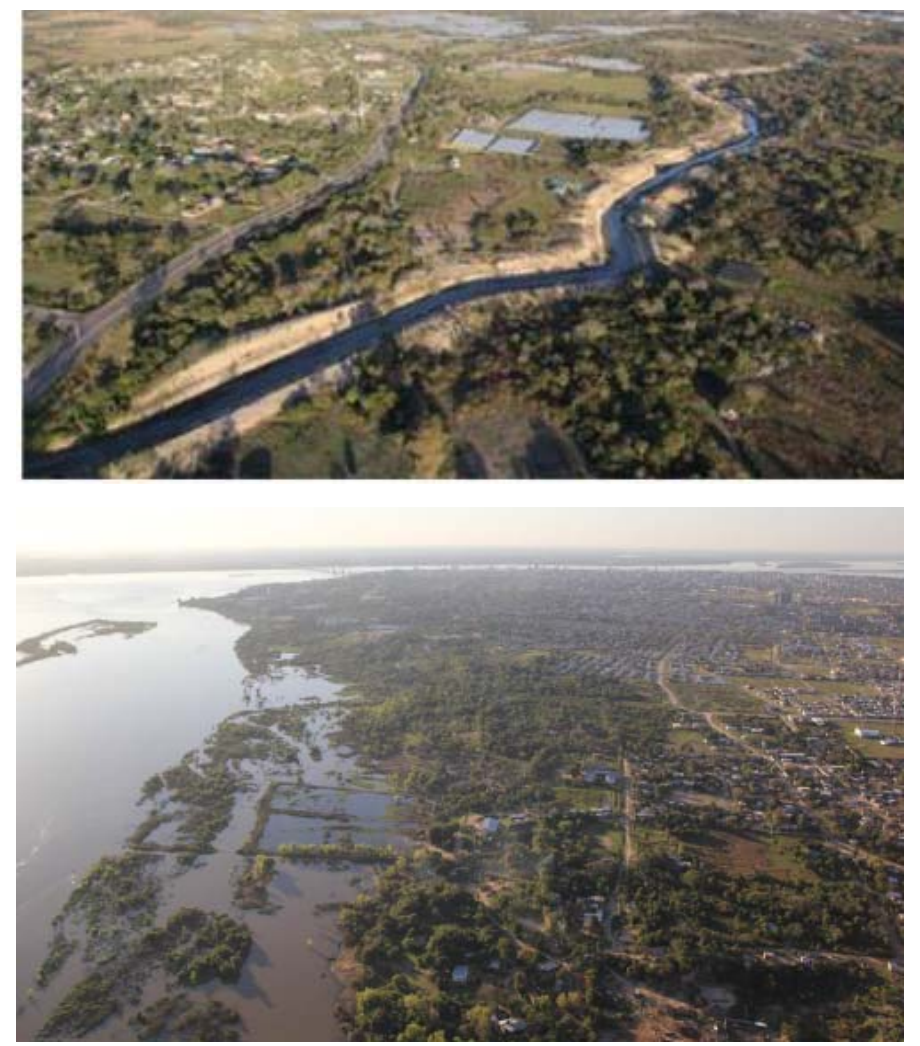

[Imagen 11. Vistas aéreas del predio. Foto Edwin Harvey, contratado para el proyecto]

Cruzando la información que surge de estas dos observaciones (matriz ambiental + matriz de grandes infraestructuras), se hacen visibles ejes que ordenan este territorio, y recintos que caracterizan espacios particulares.

Con los elementos señalados en la iimagen 13 se propone una nueva lectura del territorio, identificando diferentes piezas, que se irán integrando a la trama urbana en diferentes momentos del proceso de urbanización. Entre la pieza que tiene como eje central al arroyo Pirayuí, al este, y la línea de costa entre el eje del electroducto, al norte, y la traza del segundo puente, al sur, se configura la mayor pieza en superficie útil de urbanización. En el espacio central de esta, y a modo de espacio central del futuro nuevo sector de la ciudad, se define un parque urbano. Con este se busca articular los grandes espacios verdes de los diferentes segmentos de la costa con el cauce del arroyo Pirayuí.

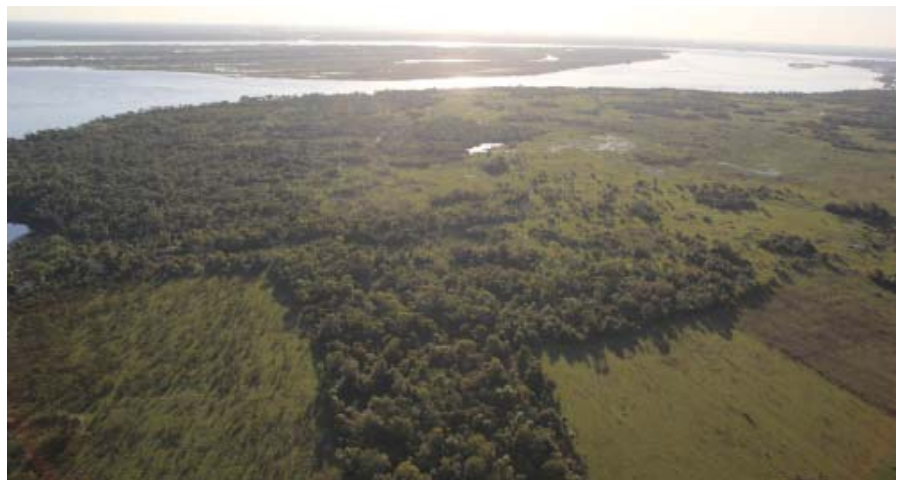

Para la definición de los patrones de subdivisión de estas grandes piezas, se buscó reproducir la estructura de chacras rectangulares de alrededor de veinte ha, que es el módulo elegido como base de actuación. Este surgió de la observación de las escalas de intervención características de la producción de suelo urbano en la ciudad de Corrientes. Para dimensionarlos, se parte del tamaño de un barrio como el área de influencia de una escuela, atendiendo a una población de alrededor de 350 familias.

La estrategia asumida apunta a proponer la posibilidad de intervenir en esta pequeña escala, asumiendo la urbanización de un barrio o de un número de manzanas que puedan conformar junto con otras una unidad mayor. El desafío consiste en garantizar la coherencia de la totalidad de la urbanización, aceptando la diversidad de las partes.

Para lograrlo, se propusieron algunos criterios de composición, tendientes a garantizar la continuidad de algunas calles y del sistema de áreas verdes, a la vez que se respetó la autonomía del trazado, permitiendo así que ciertos rasgos particulares fortalezcan la identidad de cada barrio.

Lasuperposición deestasleyes de generación de una geometría general que sostenga la particularidad de los diferentes trazados barriales se superpone con las características de los diferentes recintos que se identificaron como unidades ambientales. El resultado es un conjunto coherente que sostiene una gran diversidad de situaciones paisajísticas y urbanas, en donde la dimensión mínima de una calle es de veinte metros, de tal manera de generar veredas anchas y forestadas.

En las unidades de gestión o chacras, a partir de la definición de determinados criterios de urbanización, se dejó abierta la posibilidad de diseño y ajuste a nivel de proyectos especiales y ejecutivos, lo que garantizará mayor calidad y diversidad de las propuestas dentro de la coherencia de conjunto. (Zonificación General. Principales usos y reservas). 

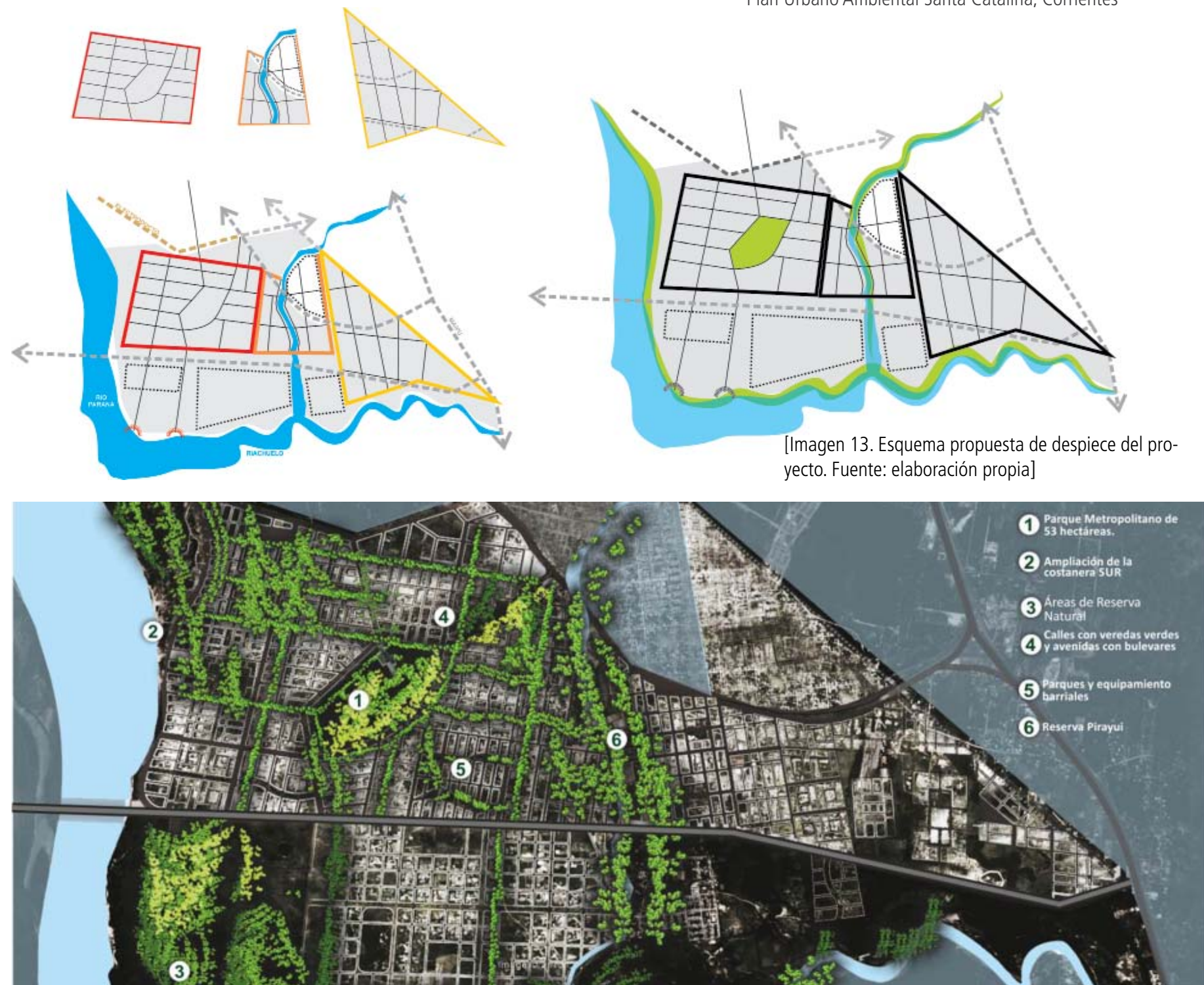

[Imagen 14. Principales reservas ambientales que incorporar a la ciudad. Fuente: elaboración propia]

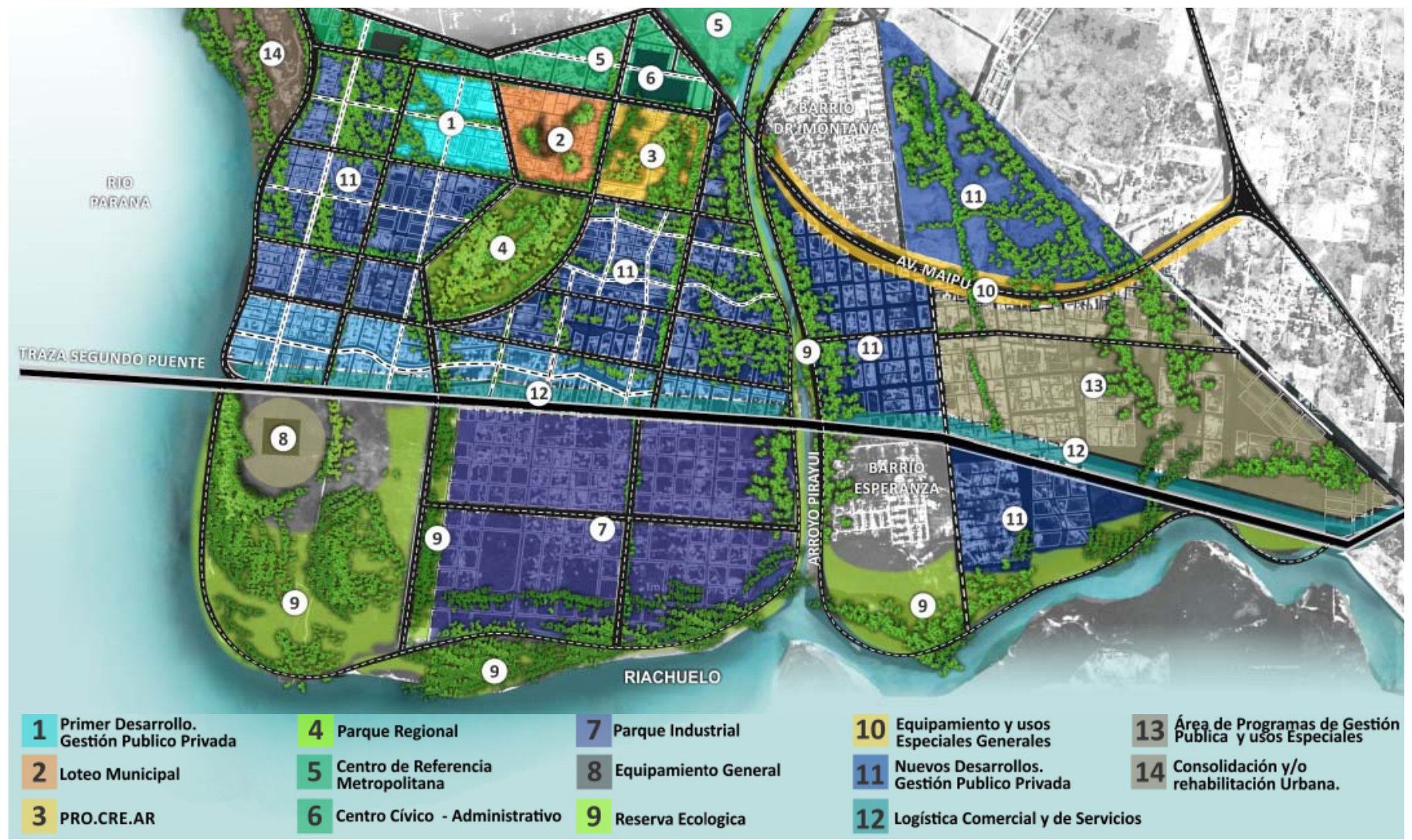

[Imagen 15. Zonificación General. Principales usos y reservas. Fuente: elaboración propia] 
"Santa Catalina puede convertirse en un capital de cambio para la obtención de mejoras en otras áreas urbanas deficitarias...".

rrientes contar con una reserva pública de suelo apto para el desarrollo futuro de la ciudad, en una localización estratégica, anticipando las directrices de ese desarrollo. A corto plazo, supone la posibilidad de introducir un contrapeso en el mercado de suelo, poniendo a disposición suelo urbano asequible, y la posibilidad de generar a partir de su venta recursos financieros propios para solventar las obras estructurales de infraestructura de conexión viaria y de provisión de servicios básicos. A largo plazo, supone la posibilidad de anticipar y orientar inversiones públicas futuras extraordinarias, como las que podrían derivarse de la construcción del segundo puente Chaco-Corrientes, reteniendo para el Estado las plusvalías, en pos de su redistribución urbana. Es decir, es una experiencia en la cual la planificación pretende ser una herramienta de política pública.

El marco normativo desarrollado constituye un instrumento básico y elemental para administrar este proceso, pero sin duda requerirá el perfeccionamiento y la ampliación de los instrumentos disponibles para garantizar la capacidad de redistribución de los beneficios que Santa Catalina pueda aportar al resto de la ciudad. Santa Catalina puede convertirse en un capital de cambio para la obtención de mejoras en otras áreas urbanas deficitarias.

El sostenimiento de los objetivos y el seguimiento del plan dependerán, indudablemente, de decisiones políticas, pero en la medida en que logre transformarse en un proyecto de conocimiento masivo y público, la propia ciudadanía podrá convertirse en garante de él. Se trata de pasos progresivos de difusión absolutamente necesarios, que el Municipio ha dado aún de manera incipiente, hasta tanto asumir su rol de propietario con ideas claras de gestión, y hasta disponer de herramientas que permitan hacerlo.

En el contexto del NEA, abre un nuevo capítulo en la gestión municipal y un precedente importante en el cambio de propiedad y de usos de grandes reservas de tierra pertenecientes al Estado Nacional. 


\section{FICHA TÉCNICA}

NOMBRE: Máster Plan Santa Catalina

EQUIPO/ DIRECCIÓN: Arq. Mgter. Julieta Repetto, Arq. Mgter. Lorena C. Sánchez./ COORDINACIÓN: Arq. Olaf Jovanovich./ EQUIPO TÉCNICO/ COORDINACIÓN GRAL.: Arq. Alfredo Garay. CONSULTORES: Dr. Lic. Lucas Ferrero, Arq. Laura Wainer, Lic. Andrés Gómez, Lic. Lucas Tissera, Arq. Anabela Roitman, Arq. Damián Rotbart, Ing. Carlos Romero, Dra. Arq. Laura Alcalá, Dr. Augusto Arduino, Arq. Fernanda Pesado, Dr. Juan José Neiff./ ASESORES SUBSECRETARÍA DE PLANIFICACIÓN DE LA INVERSIÓN PÚBLICA: Dra. Miriam Santos, Arq. Carla Galeota, Diego Vesciunas/ IMÁGENES Y GRÁFICOS: Arq. Carolina Balangero, Arq. Mario Rivas, José Novo.

\section{INSTITUCIONES INVOLUCRADAS}

Municipalidad de la Ciudad de Corrientes. / Subsecretaría de Planificación de la Inversión Pública, Ministerio de Planificación Federal, Inversión Pública y Servicios./ CONICET.

\section{MODELO DE GESTIÓN IMPLEMENTADO}

Fideicomiso de Administración y Financiero Santa Catalina./ Año de proyecto: 2012 / 2013/ Ubicación (tipo de zona)/ Suroeste del Departamento Capital de la Ciudad de Corrientes. La parte norte del predio se encuentra comprendida dentro de la Zona de Reserva Urbana, según Código de Planeamiento de la Ciudad, y el resto dentro de Área Rural./ Extensión de la superficie: 2387 ha.

\section{CARACTERÍSTICAS FÍSICAS DEL ÁREA DE IMPLANTACIÓN}

Corrientes, en general, y Santa Catalina, en particular, poseen paisajes de linaje fluvial del Paraná, que en muchos sectores se encuentran aún bien conservados. Con una topografía medianamente uniforme, su nivel altimétrico en ocasiones sobrepasa la media de la ciudad consolidada, ubicándose en una situación privilegiada. Se encuentra dentro del corredor bioceánico e hidrovía Paraná-Paraguay, y se ve favorecida en términos de conectividad con países que intervienen activamente en la comercialización e intercambio de productos y de servicios. Corrientes se encuentra en el sistema interconectado nacional de energía, que le permite utilizar y, en el futuro, ampliar la utilización de la energía proveniente de distintos emplazamientos hidroeléctricos. 